comment that histologically the majority of glioma retinae appear to originate in the inner nuclear layer, a few in the ganglion cell and nerve fibre layer.

They discuss the formation of rosettes as attempts at partial differentiation to a form imitating more or less closely the primitive epithelium of the neural tube rather than the highly specialized rod and cone cells. For what it is worth they agree that rosettes are sometimes found in metastatic growths and in medullo-blastomas of the central nervous system and in neuro-blastomas of the sympathetic nervous system.

The authors describe pseudo-rosettes which are aggregations of compressed glioma cells arranged around a blood vessel and sometimes bordering a clear space.

They classify glioma retinae into three types based on cytology, the degree of undifferentiation of the cells and thus the grade of malignancy. These are (1) Retino-blastoma, highly undifferentiated: (2) Neuro-epithelioma, showing slight or partial differentiation as indicated by rosettes or pseudo-rosettes. (3) Astrocytoma, well differentiated neoplasms. Rare in the retina. The cells nearly resemble normal astrocytes. The authors comment on the serious prognosis of the retino-blastoma type but in a large proportion of these cases described by them the optic nerve and extra-ocular tissues had already been invaded.

H. B. Stallard.

\title{
CORRESPONDENCE
}

\section{ANOMALOUS PUPIL REACTIONS}

To the Editors of The British Journal of Ophthalmology.

DeAR SIRS,-It is clear that Cases 1 and 2 reported by Dr. Edelston in his interesting paper entitled "Anomalous Pupil Reactions," Brit. Jl. of Ophthal., November, 1942, were suffering from tabes, but since he does not tell us whether the reaction to near vision was present or not, some doubt-may exist in the minds of those who have read his paper as to whether a diagnosis of tabetic pupil is justified.

For the purpose of this comment however, I assume that the reaction to near vision was present and that the anomalous reaction, viz., dilatation instead of constriction to light, was tabetic in origin.

This paradoxical reaction has been described as occasionally ocçurring in tabetic pupils, and at rare intervals has been noticed by ophthalmologists and neurologists. As far as I know this 
paradox has never received an explanation - the following attempt is offered.

- It is not always realised that the tabetic pupil is caused by a lesion which destroys two different kinds of nerve fibres. Its small size and the facts that it does not dilate in the dark or to atropine, point to paralysis of the dilator reflex arc which is supplied by sympathetic fibres ( $f f$. Horner's syndrome). That it does not react to light shows that the light reflex arc is damaged, which is parasympathetic in origin. The near vision reflex arc is also parasympathetic, but since it is unaffected and its fibres are anatomically far removed from the dilator and light reflex arcs, no further mention of it is necessary. We may therefore define the tabetic pupil as a total sympathetic and a partial parasympathetic iridoplegia. Starting from the retina the afferent limbs of the dilator and light reflex arcs travel in the optic nerve and tract to reach the mid-brain via the brachium of the superior colliculus-they then diverge from one another, the dilator fibres passing downwards to the cilio-spinal centre in the spinal cord, the constrictor fibres passing inwards to Edinger-Westphal's nucleus.

The two sets of fibres therefore are in contact with one another only in the optic nerve, tract, and in the brachium. Since both are damaged in the Argyll Robertson pupil syndrome, the lesion causing it must either be in the optic nerve, tract, or brachium. 'The optic nerve is unlikely because of the frequent absence of optic atrophy. The tract equally so because of the absence of homonymous hemianopia.

This leaves us with the brachium of the superior colliculus in which the sympathetic and parasympathetic fibres are in close proximity unguarded by visual fibres. It is here therefore that the lesion causing the tabetic pupil is postulated.

Furthermore it is conceivable that in this situation a few fibres of the light reflex arc may succeed in regenerating, and instead of joining up with parasympathetic fibres pass into sympathetic fibres. Should this have taken place the stimulus of light wauld activate sympathetic fibres causing dilation of the pupil instead of constriction.

In support of this suggestion I would point out that there is an analogous condition which is not uncommon-I refer to the fact that after a severe Bell's palsy certain flavours cause a secretion of tears. The facial nerve contains autonomic fibres subserving both taste and lacrymal secretion. It has been suggested that some of the afferent taste fibres in regenerating have joined up with some of the efferent lacrymal fibres so causing certain flavours to produce tears.

All of us will agree with Dr. Edelston in his diagnosis that Case 3 is an instance of the syndrome usually called myotonic pupil. 
Clinically it is important to be able to differentiate this condition from tabes. As a rule this is very easy for the tabetic pupil is nearly always bilateral, the myotonic nearly always mono-lateral. In tabes when the knee jerks and ankle jerks are absent there is always Rhombergism. In the myotonic pupil syndrome even when both pupils are affected, and the knee jerks and ankle jerks are absent, neither Rhombergism nor sensory symptoms are present.

It has been suggested that since, as far as the pupillary reactions are concerned, the lesion is probably in the ciliary ganglion, "Ciliary Ganglion Irido-plegia" is a better name for the syndrome (Brit. $\mathrm{Jl}$. of Ophthal., February, 1942).

Yours faithfully,

Percival W. Leathart.

31, RODNEY STREET,

LIVERPOOL, 1.

November 15, 1942.

\section{PRONTOSIL SOLUBLE IN CONJUNCTIVITIS}

To the Editors of THE BRITISH JOURNAL OF OPHTHALMOLOGY.

DEAR SiRs,-The article by J. Horton Young, R.A.M.C., which appeared in the June issue of the Journal is full of interest.

I have already sent to the Medical Journal of Australia an account of the value of prontosil soluble $2 \frac{1}{2}$ per cent. in conjunctivitis, and the use of finely strained sulphanilamide in cod liver oil in corneal ulceration. But as indicated in that sketch the puzzle is to. know what the prontosil really does.

In the most severe, and to me unique case of conjunctivitis I have ever seen it was most effective, but two thorough bacteriological investigations showed nothing but diphtheroid organisms and some polymorph cells.

I refer also to the experimental work of Robson and Scott published in Nature, April 22, 1942.

Apparently prontosil is absorbed by the conjunctiva rapidly, and effects some favourable change. As indicated, I have been using it in a severe case of spring catarrh with success. It seemed to me that administration orally was not logical as one only hopes that it will reach the eye, whereas the local administration deals directly with the diseased tissue and in any event administration by the mouth in a protracted case would be dangerous.

I have found, as J. Horton Young has found, that it does not irritate, nor does it interfere with any other drops that may be used. About its use in deep infection of the eye I have no experience at present. It is too much to expect that we have found a universal 